\title{
Química nuclear: Aplicaciones de salud en Honduras
}

César Mauricio Urbizo Vivas *

\section{RESUMEN}

La química nuclear es una rama de la ciencia que tiene sus orígenes a finales del siglo XIX y goza de múltiples aplicaciones a nivel mundial. El presente trabajo pretende ilustrar al lector cuáles son los principios básicos que fundamentan las teorías nucleares. Muestra algunas de las aplicaciones de salud más destacadas en Honduras y la legislación vigente que las rige. Se presenta una revisión bibliográfica sobre los últimos avances referidos a las moléculas radioactivas con aplicaciones terapéuticas.

Palabras Clave: Isótopos, radioactividad, vida media, fusión, fisión, Medicina nuclear, radioinmunoterapia, anticuerpos monoclonales, gamma cámara

\section{ABSTRACT}

Nuclear chemistry is a branch of science that has its origins in the late nineteenth century and has multiple applications worldwide. The present work illustrates the reader about which are the basic principles of its theories. It shows some of its most prominent health uses in Honduras and the legislation which governs them. And present a literature review on the latest advances in radioactive molecules with therapeutic applications.

Key Words: Isotope, radioactivity, half life, fusion, fision, nuclear medicine, radioimmunotherapy, monoclonal antibodies, gamma chamber

Universidad Nacional Autónoma de Honduras. Facultad de Química y Farmacia. Escuela de Química y

Farmacia 


\section{INTRODUCCIÓN}

La química general es la responsable del análisis de los compuestos químicos, sus propiedades, orbitales electrónicos y su capacidad de hibridación para formar nuevos enlaces con otros átomos o moléculas. La Química Nuclear es la rama de la físicoquímica encargada del estudio de los núcleos atómicos, sus propiedades y reacciones.

A nivel general, el estudio de la química se limita a un nivel electrónico. A partir de su configuración electrónica, se estudia cómo reaccionan los átomos y moléculas cuando forman el octeto (ocho electrones en su última capa de valencia), así se confiere mayor estabilidad y la obtención nuevos productos. Esta disciplina devela una nueva identidad de los átomos. Los núcleos también pueden reaccionar como lo hacen los electrones, pero regidos bajo reglas nucleares. La química nuclear estudia este comportamiento particular de la naturaleza que se dá bajo ciertas condiciones.

\section{HISTORIA}

En 1885 Henri Becquerel descubrió que las sales de Uranio emiten radiación que impresiona las placas fotográficas. Posteriormente se demostró que esta radiación puede ionizar el aire. La misma se emite desde el elemento o sus sales, y no es alterada por la temperatura ni por la fuente de Uranio.

En 1898 Marie y Pierre Curie encontraron que el mineral Pecblenda (principalmente Uranio $\mathrm{U}_{3} \mathrm{O}_{8}$ ) exhibía un comportamiento más pronunciado que el Uranio mismo, lo que sugería la presencia de elementos más activos que éste, y finalmente madame Curie aisló dos nuevos elementos de dicho mineral: el Polonio y el Radio. Poco después se hizo el descubrimiento del Torio y del Actinio. Hasta la fecha se han descubierto más de 30 elementos naturales que emiten estas radiaciones y otros que se obtienen a partir de procedimientos artificiales que se describirán más adelante.

\section{RADIOACTIVIDAD}

La emisión espontánea de radiación por algunos átomos se denomina Radioactividad. Alos elementos o compuestos que presentan este comportamiento se les denomina Radioactivos. Los isótopos son los átomos de un mismo elemento; pero contienen distinta masa. Los diferentes isótopos de un átomo suelen tener propiedades físicas diferentes (temperatura de fusión, temperatura de ebullición, 
velocidad de difusión, y en algunos casos radioactividad). Un ejemplo de ello son los dos isótopos comunes de Uranio: ${ }_{92} \mathrm{U}^{235}$ no radioactivo y el ${ }_{92} \mathrm{U}^{238}$ radioactivo, ambos con 92 protones en su núcleo.

\section{NATURALEZA DE LAS RADIACIONES}

Las radiaciones emitidas por los elementos radioactivos y sus sales consisten en 3 tipos: alfa (á), beta (â) y gamma (ã).

\section{Radiación alfa ( $\alpha)$}

El estudio de los rayos á en un campo magnético ha revelado que constan de partículas materiales con una masa y carga de cuatro, y dos veces respectivamente la del protón; es decir, que resulta idéntica a la de los núcleos de Helio $\left(\mathrm{He}^{4}\right.$, dos protones unidos con dos neutrones), comprobado experimentalmente. Estas partículas tienen la capacidad de ionizar los gases y de penetrar la materia, sólo pueden viajar centímetros en el aire, o centésimas de milímetros a través de sólidos. Su velocidad inicial es muy grande, varía desde $1-2 \times 10^{9} \mathrm{~cm} / \mathrm{s}$ contra $3 \times$ $10^{10} \mathrm{~cm} / \mathrm{s}$ para la velocidad de la luz.

\section{Radiación beta $(\beta)$}

La radiación â consiste en partículas de masa mucho menor y carga negativa. Los rayos â son haces de electrones que pueden ionizar los gases unas 1/100 veces menor que la radiación á. Esto se debe a la masa reducida. Los rayos beta también impresionan placas fotográficas y poseen la cualidad de penetrar la materia; pero con un poder de penetración aproximadamente 100 veces mayor que las partículas á. En algunas ocasiones dependiendo de la fuente de emisión. La velocidad de los electrones es aproximadamente la de la luz. La desintegración â puede clasificarse en dos tipos: beta menos $\left(\beta^{-}\right.$, beta mas $\left(\beta^{+}\right)$

\section{Desintegración $\beta^{-}$}

La emisión â?implica la transformación de un neutrón en un protón, un electrón y una tercera partícula llamada antineutrino (ã). El antineutrino es la antipartícula del neutrino; ambas tienen masa y carga cero, por lo que producen muy pocos efectos observables al pasar por la materia. La naturaleza de esta tercera partícula es de tipo antimateria.

n

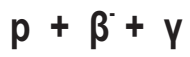

\section{Desintegración $\beta^{+}$(Positrón)}


La emisión â+ surge por la transformación de un protón en un neutrón, un electrón y un neutrino electrón.

$$
\mathrm{p} \quad \mathrm{n}+\beta^{+}+\mathrm{Y}_{\mathrm{e}}
$$

\section{Radiación Gamma (v)}

Las radiaciones gamma (ã) no son afectadas por los campos eléctricos 0 magnéticos. Se comportan totalmente como una radiación electromagnética. Su longitud de onda es del orden de $10^{-8}-10^{-11} \mathrm{~cm}$ de longitud, por esta razón se encuentra en uno de los extremos del espectro de radiaciones. En ciertas bibliografías se describen estas ondas como rayos cósmicos, debido a que también son producidas por algunos fenómenos de cuerpos celestes, por ejemplo en explosiones de estrellas, agujeros negros, etc.

Al igual que las radiaciones anteriores éstas impresionan placas fotográficas, ionizan los gases y penetran la materia. Su poder de penetración es de 10 a 100 veces la de los rayos â; pero su capacidad ionizante es mucho más débil. Un elemento que emite radiación ã no transmuta, solo cambia de un estado excitado a otro de menor excitación.

\section{DESINTEGRACIÓN RADIOACTIVA}

Que un elemento o compuesto inestable emita cualquier tipo de radiación implica la pérdida de un componente en su interior; ésto se debe a la búsqueda de una configuración más estable. Esta modificación en su configuración electrónica trae como consecuencia la transmutación o cambio de un elemento a otro. En 1903 Rutherford y Soddy establecieron su teoría de la desintegración radioactiva.

Estos autores proponen que por la emisión de una partícula á, la masa debe disminuir en 4 unidades y la carga baja en 2 . Esto es debido a las características de la radiación á, 4 unidades de masa y 2 unidades de cargas positivas. La emisión de una partícula â provoca el aumento en una unidad. Recuérdese que la desintegración â? proviene de la desintegración de un neutrón que emite una partícula â?, un protón y un antineutrino.

Por ejemplo, en la desintegración del ${ }_{92} \mathrm{U}^{238}$ (isótopo de Uranio con una masa de 238 y una carga de 92) después de liberar una partícula á, su masa disminuye en 4 unidades y su carga baja a 90. Luego, producto del desbalance de cargas, el átomo 
libera 2 electrones para equilibrarlas y de esta manera obtener ${ }_{90} \mathrm{Th}^{234}$, un isótopo del Torio de 234 unidades de masa y carga igual a 90. Cuando el ${ }_{90} \mathrm{Th}^{234}$ emite una partícula â se forma el ${ }_{91} \mathrm{~Pa}^{234}$, un isótopo del Proactinio. Este producto posee una carga igual a 91; pero de igual masa que su progenitor. Ésto se debe a que la masa de la partícula â es extremadamente pequeña.

La radiación ã surge de los reordenamientos de energía dentro del núcleo, después de la expulsión de una partícula á o â. Si este reagrupamiento lleva a una energía potencial más baja, el exceso se desprende como un quantum energético de la frecuencia del rayo ã. No todas las transformaciones van acompañadas por la emisión de rayos ã.

Todo este comportamiento es descrito por la Ley del Desplazamiento de FajansSoddy-Russell, que establece que si un núcleo emite una partícula á, su número atómico disminuye en dos unidades y el nuevo elemento se desplaza dos posiciones hacia la izquierda en la tabla periódica. Por otra parte, cuando el núcleo original emite una partícula â, el número atómico aumenta uno, de aquí que el producto se desplace un lugar hacia la derecha en dicha tabla.

Los alquimistas por siglos buscaron la "Piedra Filosofal" y la transformación de todo lo que tocaba en oro. Con los últimos adelantos en química nuclear la idea resulta teóricamente probable.

\section{RADIOACTIVIDAD ARTIFICIAL}

Algunos isótopos inestables bajo ciertas condiciones pueden exhibir el fenómeno de la radioactividad. Éstos bajo la influencia de un bombardeo previo emiten lo que se conoce como Radioactividad Artificial o Inducida, que responde bajo las mismas reglas de la radioactividad natural y además puede darse en núcleos ligeros.

\section{VIDAMEDIA}

El tiempo de desintegración o existencia de los núcleos radioactivos responde a una función exponencial decreciente. La siguiente ecuación describe el tiempo requerido para la desintegración de la mitad de los núcleos radioactivos.

$$
\mathrm{T}_{1 / 2}=\ln 2 / \mathrm{k}
$$

El valor de la constante de proporcionalidad k es característico de cada especie radioactiva. El modelo matemático que a continuación se presenta, muestra la 
relación temporal existente entre el número de núcleos iniciales y finales, regulados por su particular constante de proporcionalidad.

$$
t=1 / k \ln N_{0} / N
$$

\section{REACCIONES NUCLEARES}

Una manera simplificada de clasificar las reacciones nucleares es describiendo la partícula de bombardeo y nombrando la partícula emitida, por ejemplo, una reacción $(p, n)$ ocurre cuando un protón bombardea un determinado núcleo y se emite un neutrón más un nuevo núcleo. Una reacción $(d, p)$ resulta del bombardeo del núcleo elegido por un deuterón ${ }_{1} \mathrm{H}^{2}$ (partícula de Deuterio acelerada) y produce un protón y otro núcleo.

Ejemplos de Tipos de Reacciones Nucleares

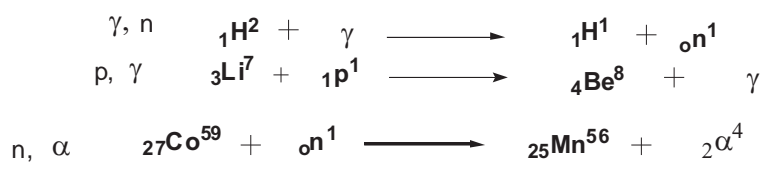

\section{Fusión Nuclear}

Como su nombre lo indica, es la unión de dos o más núcleos ligeros que al fusionarse forman un núcleo más pesado.

La reacción exotérmica que se dá en las estrellas es de tipo Termonuclear protónprotón $(p, p)$, y se describe en las siguientes ecuaciones:

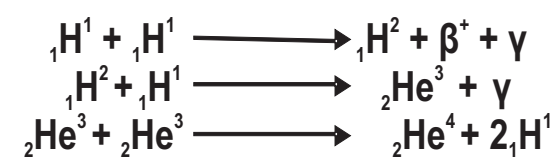

\section{Fisión Nuclear}

La fisión nuclear ocurre cuando por bombardeo de partículas se fracciona un núcleo pesado en fragmentos considerablemente más ligeros. Un núcleo fisionable es el ${ }_{92} \mathrm{U}^{235}$ que se describe con la siguiente reacción:

${ }_{92} \mathbf{U}^{235}+{ }_{0} \mathbf{n}^{1} \longrightarrow{ }_{92} \mathbf{U}^{236} \longrightarrow{ }_{56} \mathbf{B a}^{144}+{ }_{36} \mathbf{K r}^{89}+{ }_{30} \mathbf{n}^{1}$ 
El uranio se encuentra en la naturaleza como mezcla isotópica, $99.28 \%$ de ${ }_{92} \mathrm{U}^{238}$, $0.71 \%$ de ${ }_{92} \mathrm{U}^{235}$ y $0.01 \%$ de ${ }_{92} \mathrm{U}^{234}$. $\mathrm{El}_{92} \mathrm{U}^{235}$ es un isótopo extremadamente escaso. La difusión de gases es un método de separación de estos isótopos; pero es un procedimiento sumamente difícil.

Una alternativa que los científicos han descubierto es la transmutación de ${ }_{92} \mathrm{U}^{238} \mathrm{a}$ ${ }_{94} \mathrm{Pu}^{239}$, un isótopo fisionable del Plutonio. El proceso de las ecuaciones nucleares se detalla a continuación.

$$
\begin{aligned}
& { }_{92} \mathrm{U}^{238}+{ }_{0} \mathrm{n}^{1} \longrightarrow{ }_{92} \mathrm{U}^{239} \\
& { }_{92} \mathrm{U}^{239} \longrightarrow{ }_{93} \mathrm{~Np}^{239}+\beta^{-} \\
& { }_{93} \mathrm{~Np}^{239} \longrightarrow{ }_{94} \mathrm{Pu}^{239}+\beta^{-}
\end{aligned}
$$

\section{UNIDADES DE MEDICIÓN}

El Gray es la unidad de dosis absorbida en el Sistema Internacional (SI). $1 \mathrm{~Gy}=1 \mathrm{~J} / \mathrm{kg}$

Existe otra unidad que goza de bastante uso, el Rad, ésta se define como: $1 \mathrm{Rad}=0.01 \mathrm{~J} / \mathrm{kg}=0.01 \mathrm{~Gy}$

Hay una unidad antigua de radioactividad que aún continúa en uso, el Curie (Ci); en honor a Pedro y Marie Curie. Un Curie corresponde a $3.7 \times 10^{10}$ desintegraciones por segundo, que son equivalentes a otra unidad: el Becquerel, o sea:

1 Curie $(\mathrm{Ci})=3.7 \times 10^{10}$ Becquerel $(\mathrm{Bq})=3.7 \times 10^{10}=3.7 \times 10^{10}$ por segundo .

Sievert (Sv), también conocida como dosis eficaz, es una unidad derivada del S! que mide la dosis de absorbida por los distintos tejidos (Gy); aplicando factores de ponderación que toman en cuenta el tipo de radiación (alfa, beta, gamma, X, neutrones).

\section{MEDICINANUCLEAR}

La medicina nuclear es la subespecialidad de las ciencias médicas que aprovecha las bondades de los fenómenos nucleares en beneficio de la salud. Las prácticas médicas nucleares más utilizadas se detallan a continuación: 
Radioterapia: tratamiento médico nuclear que consiste en el bombardeo de tejidos vivos de radiación gamma emitida por una fuente externa. Este procedimiento lo realiza una Unidad de Cobalto -60.

Braquiterapia: terapia radioactiva que se basa en ubicar la fuente de radiación directamente sobre la zona de interés.

Yodoterapia radioactiva, una alternativa para tratar el hipertiroidismo, es la terapia con yodo radioactivo (I-131). Se administra por vía oral, usualmente en forma de una cápsula pequeña que se toma una sola vez y se absorbe rápidamente en el organismo a través del intestino. Viaja por el torrente sanguíneo hasta la tiroides, donde es absorbido. La glándula capta el yodo sea radioactivo o no.

El tratamiento destruirá de forma selectiva el tejido de la tiroides para evitar que la tiroides produzca hormonas tiroideas en exceso. Lo que no es retenido en la glándula tiroides se excretará por la orina 24 horas después de tomar la dosis de yodo. La mayoría de los pacientes que son tratados con yodo radiactivo desarrollan hipotiroidismo, que puede ser tratado fácilmente con un suplemento de hormona tiroidea que se toma una vez al día o seguirán teniendo hipertiroidismo; generalmente menos severo que antes. El I-131 emite rayos gama, similares a los rayos- $x$. Estos rayos gamma pueden ser detectados por detectores especiales.

Terapia con Radiofármacos, utiliza productos farmacéuticos que poseen incorporado en su estructura algún componente radioactivo.

\section{MEDICAMENTOS RADIOACTIVOS DE USO DIAGNÓSTICO}

Poseen 2 propósitos:

\section{Exploraciones Diagnósticas con Imagen}

Se trata de exploraciones gammagráficas. Se aprovecha la afinidad del radiofármaco empleado por un determinado órgano (el órgano diana), donde tiende a acumularse el trazador. Con la instrumentación adecuada, puede captarse la radiación emitida por el radiofármaco, acumulado en el órgano diana desde el exterior del cuerpo.

La radiación captada es transformada en imágenes de las que se obtiene información morfológica y funcional. Las exploraciones gammagráficas representan más del $90 \%$ de las exploraciones realizadas. 


\section{Exploraciones Diagnósticas sin Imagen}

Se denominan también test diagnóstico in vivo. Se aprovecha el comportamiento del radiofármaco en un determinado comportamiento biológico para poder detectarlo y cuantificarlo por la radiación que emite, obteniendo así información diagnóstica sobre el órgano o la función estudiada, pero con ausencia de imágenes.

Ejemplo de este tipo de exploraciones son: la captación tiroidea de radioyodo, la absorción de vitamina $\mathrm{B}_{12}$ marcada con isotopos radioactivos de cobalto y la determinación de volumen sanguíneo o plasmático.

\section{TIPOS DERADIOFÁRMACOS DEUSO DIAGNÓSTICO}

Los radiofármacos se clasifican en 3 categorías:

\section{Radiofármacos Listos para su Uso}

Los Radiofármacos Listos para su Uso están garantizados por el fabricante y son presentados en viales multidosis. Las operaciones de preparación son procesos simples similares a medicamentos convencionales (fraccionamiento, dilución, reconstitución, etc.), que deben realizarse en condiciones higiénicas.

\section{Radiofármacos Obtenidos de Reactores Generadores}

Son la mayoría de los radiofármacos utilizados en medicina nuclear. Ciertos radioisótopos empleados en la actualidad poseen una vida media corta; lo que obliga a la preparación del radiofármaco antes de su administración. La preparación debe realizarse siguiendo instrucciones particulares por el fabricante del generador radiactivo. Es necesario garantizar el radiofármaco final que se administraráal paciente.

\section{Otros Radiofármacos}

Otro tipo de radiofármacos son los autólogos, se basan en proteínas o células sanguíneas del propio paciente para su marcaje con un radioisótopo. Deben prepararse en condiciones de esterilidad. Frecuentemente, se necesita prepararlos con procedimientos abiertos donde los riesgos bacterianos sean altos. Debe garantizarse las características del producto final. 


\section{MEDICAMENTOS RADIOACTIVOS DE USO TERAPÉUTICO}

Los Medicamentos Radioactivos de uso Terapéutico son productos radiofarmacéuticos que aprovechan su afinidad por un determinado órgano para transportar hasta ese órgano diana la suficiente radioactividad como para dar dosis terapéuticas de radiación, procurando que ésta sea reducida en otros órganos.

La Radioinmunoterapia es un novedoso tratamiento antineoplásico de carácter dual. Combina la actividad de un anticuerpo monoclonal con los efectos terapéuticos de las ondas radioactivas emitidas por un radioisótopo. Ejemplo de este tipo de radiofármacos son los Anticuerpos Monoclonales Radioactivos. Proteínas sintéticas producidas en laboratorios que reconocen y se unen a un sitio target para atacar las células oncológicas. Los nombres genéricos de estos medicamentos siempre terminan en "mab". Se debe al término inglés para designar a los anticuerpos monoclonales: monoclonal anti-bodies.

\section{IBRITUMOMAB}

El Ibritumomab es un radioanticuerpo que emplea el Ytrio-90 como fuente de radiactividad. Actúa independientemente de la localización del linfoma. Ha sido aprobado asignado para pacientes adultos con LINFOMA NO HODGKIN tipo B antígeno CD20, cuando el rituximab (anticuerpo monoclonal no radioactivo) ya no surte efecto. Permite la destrucción máxima de las células del linfoma minimizando la exposición general del paciente a la radiación. No pueden ser utilizados con quimioterapia ya que reduce los recuentos sanguíneos, disminuye la coagulación sanguínea, causa debilidad y fatiga, lo que puede aumentar el riesgo de infecciones, sangrados u otros problemas.

\section{TOSITUMOMAB}

El Tositumomab es una nueva forma de anticuerpo monoclonal que emplea Yodo131 como radioisótopo. Se ha recomendado para el tratamiento de LINFOMAS NO HODGKIN. Las pruebas demuestran que es más poderoso que el rituximab. Su vía de administración es intravenosa pero de difícil manipulación debido a la radioactividad que presenta.

El Radioanticuerpo va dirigido al antígeno CD20 en células B sanas y malignas. Las células madre (células nuevas en la médula ósea que se desarrollarán en los distintos tipos de células) no poseen el antígeno CD20. Ésto permite que las células sanas puedan regenerarse después del tratamiento. 


\section{QUÍMICANUCLEAREN HONDURAS}

La implementación de la química nuclear en Honduras es relativamente nueva; por tal razón sus aplicaciones en salud, industria, agricultura, investigación, docencia y otras áreas, por el momento, son limitadas. El sábado 14 de noviembre de 2009 fue publicada en el Diario Oficial "La Gaceta" la Ley Sobre Actividades Nucleares y Seguridad Radiológica, que regula toda práctica nuclear de uso exclusivamente pacífico desarrollada en el país coherente con el acuerdo suscrito por Honduras con la Agencia Internacional de EnergíaAtómica (AIEA).

La ley y sus reglamentos permiten el apoyo significativo por parte de países y organizaciones internacionales, anuentes a colaborar con asistencia técnica en materia de asuntos nucleares y de esta manera acelerar el desarrollo económico y social del país.

El Artículo 4.- de la presente ley designa a la Secretaría de Estado en los Despachos de Recursos Naturales y Ambiente (SERNA), a través de la Dirección General de Energía, para la regulación y control que se deriva de los reglamentos respectivos.

El campo de aplicación más desarrollado en Honduras es la Medicina Nuclear. El Instituto Hondureño de Seguridad Social (IHSS) desea innovar en Honduras con el uso de Ibritumomab y Tositumomab como alternativa terapéutica antineoplásica. Instituciones de salud establecidas en Tegucigalpa que realizan algunas prácticas nucleares mencionadas anteriormente son:

\section{Centro de Cáncer Emma Romero de Callejas}

En Marzo de 1991, la Asociación Hondureña de Lucha contra el Cáncer (AHLCANCER) creó El Centro de Cáncer Emma Romero de Callejas (CCERC), institución privada sin fines de lucro. Inició el servicio de Radioterapia con una Unidad de Cobalto -60 para el tratamiento de tumores malignos. Se instaló para este fin una gamma cámara. El CCERC presta los servicios de Yodoterapia para pacientes con cáncer de tiroides e hipertiroidismo. En Radiodiagnóstico se realizan estudios sobre funcionalidad de patologías en órganos como huesos, riñones, pulmones, etc.

En 2005 se implementó el servicio de Braquiterapia. Se brinda tratamiento contra tumores ginecológicos con baja tasa de dosis de Cesio -137. La institución cuenta con quirófanos especializados y habitaciones blindadas para el apropiado manejo y 
realización de estas prácticas. Se cuenta con un cuarto caliente o gammateca para calibración y depósito de las fuentes radiactivas. El control de calidad del equipo es supervisado por SERNA bajo los lineamientos AIEA.

\section{Hospital General San Felipe}

Fue fundado el 27 de Agosto de 1882, considerado el primer centro asistencial. Comenzó las actividades nucleares de Radioterapia en 1990 con una unidad de Cobalto -60. En la actualidad cuenta con 2 unidades y presta el servicio de atención público más importante de Honduras. La AIEA asociada con la OPS/OMS apoya la evaluación del servicio de radioterapia en este hospital. En la modalidad de Braquiterapia cuenta con una unidad de mediana tasa de dosis de Cesio -137 que aún no está en funcionamiento por falta de un quirófano.

\section{AGRADECIMIENTOS}

En primer lugar a Dios, creador del universo y de las leyes físicas, por permitirme concluir este trabajo.

A los siguientes profesionales distinguidos, gracias por sus constantes aportes y valiosa ayuda:

M.Sc. Filiberto Rodríguez (Físico-Médico) Sub-Director Técnico CCERC; Coordinador del Técnico en Radiotecnología (UNAH)

Oscar A. Urbizo Vivas - Traducción

Especialmente a mi esposa Dra. Marilyn Guevara Peña, a mis hijos Mauricio Javier Urbizo Guevara y César Darío Urbizo por sus muestras de cariño, comprensión y motivación.

Fue un honor haber trabajado con ustedes

\section{CONCLUSIONES}

- En Honduras, la tecnología nuclear más importante se encuentra en el campo de la salud, pero existen otras pequeñas aplicaciones de radioactividad aún no desarrolladas.

- La Ley Sobre Actividades Nucleares y Seguridad Radiológica controla toda 
actividad nuclear pacífica; permitiendo una mayor colaboración de países u organismos con mejor conocimiento y experiencia en el campo. La Secretaría de Recursos Naturales y Ambiente (SERNA) es la autoridad reguladora a través de la Dirección General de Energía.

- A nivel mundial, ya se desarrollaron los radioanticuerpos, medicamentos radioactivos de última generación para el combate de Linfomas No Hodgkin tipo B. De las moléculas proteicas sintéticas diseñadas existen 2 en el mercado Ibritumomab y Tositumomab. En Honduras se realizan las gestiones pertinentes para su importación.

\section{BIBLIOGRAFÍA}

Diario Oficial "La Gaceta" de la República de Honduras No.32,062 Sábado 14 de Noviembre de 2009.

http://ccerc.org/index.aspx?Type=C\&cod=16

http://fisikmedik.wordpress.com/

http://www.chemocare.com/es/bio_es/ibritumomabtiuxetan_ES.asp

http://www.chemocare.com/es/bio_es/tositumomab_ES.asp

http://www.radiologyinfo.org/sp/info.cfm?pg=gennuclear

http://www.sievert-system.org/WebMasters/sp/mesure.html

Mallol, Jesús. Manual de Radiofarmacia. España: Ediciones Díaz de Santos; 2008.

Mallol, Jesús. Medicamentos Radioactivos Radiofármacos y Productos

Radiofarmacéuticos. España: Ediciones Díaz de Santos; 1995

Maron, Samuel H. y Prutton, Carl F. Fundamentos de Fisicoquímica. Última Edición. México: Limusa; 2006

Sears, Francis W., Zemansky, Mark W., Young, Hugh D. y Freedman, Roger A.

Física Universitaria. Vol. 2. Undécima Edición. México: Pearson; 2004 